\title{
Asymmetric synthesis of multi-substituted triptycenes via enantioselective alkynylation of 1,5-dibromoanthracene-9,10-dione
}

\author{
Takanori Shibata $^{*}$,a,b and Yuri Kamimura ${ }^{a}$ \\ ${ }^{a}$ Department of Chemistry and Biochemistry, School of Advanced Science and Engineering, Waseda University, Shinjuku, Tokyo, 169-8555, Japan \\ ${ }^{b}$ JST, ACT-C, 4-1-8 Honcho, Kawaguchi, Saitama 332-0012, Japan \\ E-mail: tshibata@waseda.jp \\ Tel\&Fax: $+81-3-5286-8098$
}

\section{ARTICLE INFO}

ABSTRACT

\section{Article history:}

Received

Received in revised form

Accepted

Available online
Enantioselective alkynylation of 1,5-dibromoanthracene-9,10-dione by alkynyllithium reagents prepared from alkynes, $n$-BuLi, and sparteine gave chiral cis-diols possessing two alkyne moieties with moderate ee. Subsequent Ru-catalyzed $[2+2+2]$ cycloaddition of the diol with alkynes afforded chiral 1,5-dibromotriptycenes.

2009 Elsevier Ltd. All rights reserved.

\section{Introduction}

Iptycene is a group of compounds that possess a bicyclo[2.2.2] octa-2,5,7-triene skeleton, in which the bridge moieties are joined by aromatic rings. Among them, the simplest one is triptycene, which is composed of three phenyl rings. Bartlett first synthesized triptycene in 1942 by a multi-step protocol. ${ }^{1}$ Wittig and Ludwig disclosed a one-step protocol of a [4+2] cycloaddition of anthracene with benzyne, ${ }^{2}$ and this has been the most conventional procedure for the preparation of triptycene derivatives. The triptycene skeleton has a rigid, threedimensional structure; therefore, it is fascinating as a key unit for making unique molecular architectures. Oki's work of triptycene-based molecular balances is a pioneering achievement, which elucidated upon non-covalent weak interactions. ${ }^{3}$ Since then, many applications of triptycenecontaining molecules have been reported. ${ }^{4}$ Diphosphine ligands, ${ }^{5}$ a molecular compass and gyroscope, ${ }^{6}$ and supermolecular system in host-guest chemistry ${ }^{7,8}$ are representative examples. Swager comprehensively studied triptycene-based polymers, which offer a new avenue for material chemistry. ${ }^{9}$

Multi-substituted triptycenes can be chiral. While an asymmetric synthesis was reported in 1962 by using resolution of diastereomeric salts, ${ }^{10}$ to the best of our knowledge an enantioselective synthesis has not been reported on. In contrast, Swager reported on a new protocol for the synthesis of triptycene derivatives using consecutive 1,2-addition of alkynyllithiums to anthraquinone along with the $[2+2+2]$ cycloaddition with alkynes. ${ }^{11}$ We considered that an enantioselective alkynylation of two carbonyl moieties of a 1,5disubstituted anthraquinone can realize an asymmetric synthesis of triptycene derivatives (Scheme 1). Herein we report a sparteine-mediated enantioselective alkynylation for the construction of chiral triptycene skeletons, followed by stereospecific $[2+2+2]$ cycloaddition.
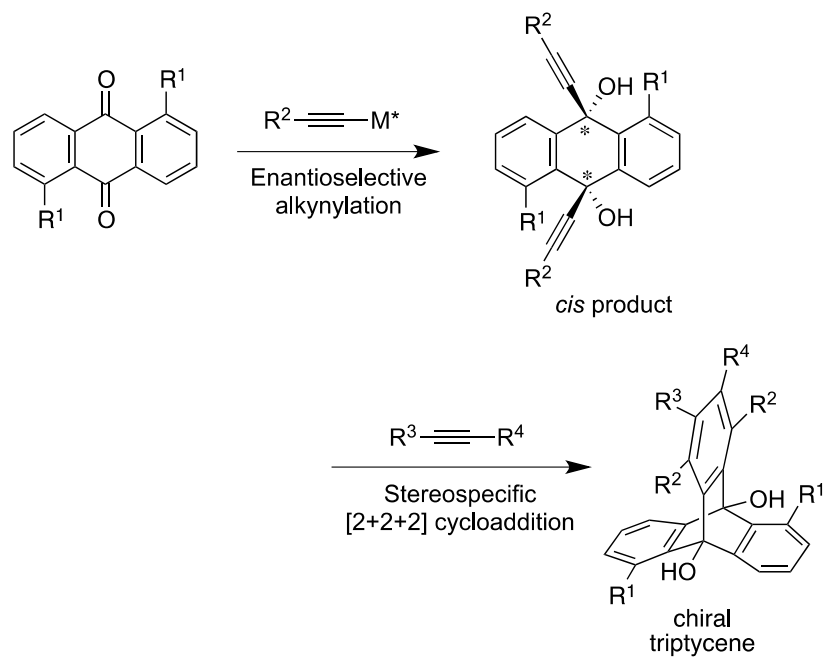

Scheme 1. Enantioselective synthesis of chiral triptycenes 


\section{Results and discussion}

We chose 1,5-dibromoanthracene-9,10-dione $\mathbf{1}$ as a model diketone for the preparation of useful chiral synthetic building blocks. The enantioselective alkynylation of 1 was examined by using a stoichiometric amount of chiral phenylethynyl lithium reagents in toluene at room temperature (Table 1). ${ }^{12-15}$ When a catalytic amount of BINOL L1 was used as the chiral ligand in THF, ${ }^{12 \mathrm{~b}}$ dialkynylated product $c i s-\mathbf{2 a}$ was obtained, but the ee was below $2 \%$ (entry 1 ). In contrast, when using more than stoichiometric amounts of (+)-sparteine L2, it induced a moderate ee of $58 \%$ (entry 2). The enantioselectivity from sparteine surrogate $\mathbf{L 3}$ was lower than sparteine (entry 3). Chiral diamine $\mathbf{L 4}$ derived from cyclohexane-1,2-diamine did not show enantioinduction but the related chiral diamine L5, which possessed bulky alkyl chains gave $35 \%$ ee (entries 4 and 5). Proline-derived L6 and 2,2'-diamino-1,1'-binaphthyl-derived L7 were not effective in this enantioselective alkynylation (entries 6 and 7). In each entry, the total yield of cis- and trans2a was excellent, but the cis-isomer was the minor product.

Table 1. Screening of ligands in consecutive and enantioselective alkynylations of diketone 1

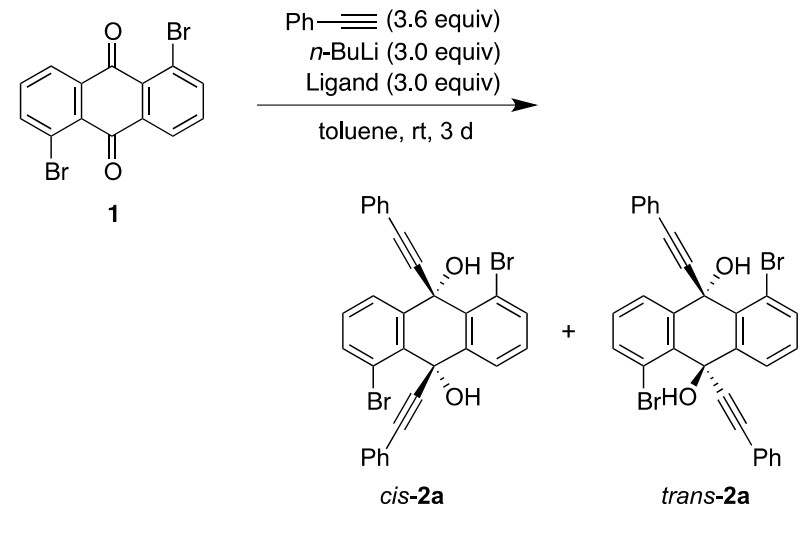

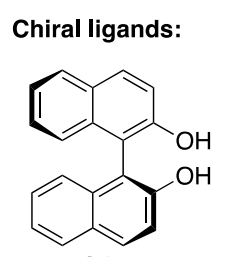

L1<smiles>[R]N(C)[C@H]1CCCC[C@H]1N([R])[Na]</smiles>

L4 $(\mathrm{R}=\mathrm{Me})$ L5 $\left(\mathrm{R}=\left(\mathrm{CH}_{2}\right)_{2} t\right.$-Bu<smiles>C1CCN2C[C@@H]3C[C@@H](CN4CCCC[C@H]34)C2C1</smiles>

(+)-sparteine (L2)

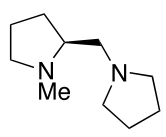

L6

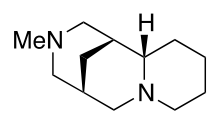

L3

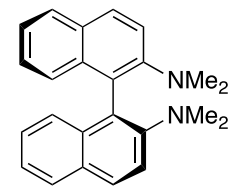

L7

\begin{tabular}{cccc}
\hline Entry & Ligand & ${\text { Yield }(\%)^{\mathrm{a}}}^{\mathrm{a}}$ & ${\text { Ee }(\%)^{\mathrm{a}}}^{\mathrm{a}}$ \\
\hline $1^{\mathrm{b}}$ & L1 & 15 & $<2$ \\
2 & L2 & 29 & 58 \\
3 & L3 & 26 & 36 \\
4 & L4 & 24 & $<2$ \\
5 & L5 & 14 & 35 \\
6 & L6 & 15 & 16 \\
7 & L7 & 23 & $<2$ \\
\hline
\end{tabular}

${ }^{\mathrm{a}}$ Yield and ee of cis-2a.

${ }^{\mathrm{b}} \mathbf{L 1}$ (20 $\mathrm{mol} \%$ ) was used in THF.
We next studied the reaction conditions using sparteine as a chiral ligand (Table 2). It was found that $s$-BuLi and $t$-BuLi gave comparable ee to those of $n$-BuLi, but the cis/trans ratio was slightly different (entries 1-3). ${ }^{16}$ A sub-stoichiometric amount of sparteine also gave enantioinduction, albeit with lower selectivity (entry 4). The solvent effect was significant on the enantioselectivity and toluene was found to give the best (entries 5-7). After screening of the reaction temperatures, a slightly better enantioselectivity was achieved at lower temperature (entries 8-10).

Table 2. Screening of reaction conditions for the enantioselective dialkynylation

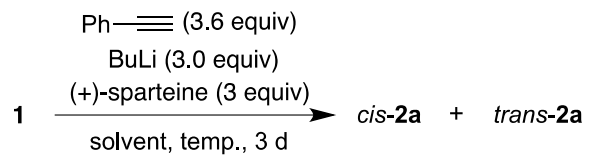

\begin{tabular}{ccccccc}
\hline Entry & BuLi & Solvent & $\begin{array}{c}\text { Temp. Yield } \\
\left({ }^{\circ} \mathrm{C}\right)\end{array}$ & $\begin{array}{c}\text { (\%) } \\
(\%\end{array}$ & cis:trans & Ee $(\%)^{\mathrm{b}}$ \\
\hline 1 & $n-\mathrm{BuLi}$ & toluene & $\mathrm{rt}$ & 97 & $1.0: 3.3$ & 58 \\
2 & $s-\mathrm{BuLi}$ & toluene & $\mathrm{rt}$ & 94 & $1.0: 4.9$ & 58 \\
3 & $t$-BuLi & toluene & $\mathrm{rt}$ & 97 & $1.0: 2.7$ & 58 \\
$4^{\mathrm{c}}$ & $n$-BuLi & toluene & $\mathrm{rt}$ & 66 & $1.0: 3.1$ & 40 \\
5 & $n-\mathrm{BuLi}$ & hexane & $\mathrm{rt}$ & 95 & $1.0: 2.6$ & 50 \\
6 & $n-\mathrm{BuLi}$ & THF & $\mathrm{rt}$ & 95 & $1.0: 3.3$ & 8 \\
7 & $n-\mathrm{BuLi}$ & ether & $\mathrm{rt}$ & $>99$ & $1.0: 3.3$ & 40 \\
8 & $n-\mathrm{BuLi}$ & toluene & 0 & 57 & $1: 0: 3.5$ & 63 \\
9 & $n-\mathrm{BuLi}$ & toluene & 10 & 94 & $1.0: 3.8$ & 64 \\
10 & $n-\mathrm{BuLi}$ & toluene & 40 & 96 & $1.0: 4.6$ & 54 \\
\hline
\end{tabular}

${ }^{a}$ Total yield of cis- and trans-isomers.

${ }^{\mathrm{b}}$ Enantiomeric excess of cis-2a.

${ }^{\mathrm{c}}$ Sparteine ( 0.5 equiv) was used.

The scope of the alkynes was investigated under the reaction conditions of entry 1 in Table 2 (Table 3). Both electrondonating and withdrawing group-substituted aryl alkynes could also be used (entries 1 and 2). In the case of 4chloroethynylbenzene, the yield of dialkynylated product $\mathbf{2 c}$ was moderate due to the formation of a mono-alkynylated product, but the ratio of the cis-isomer increased and the enantioselectivity was almost the same as that of phenylacetylene. 1-Hexyne also gave the corresponding product $\mathbf{2 d}$ and the ee of $c i$ s-isomer was moderate (entry 3 ).

Table 3. Scope of alkynes in the enantioselective dialkynylation

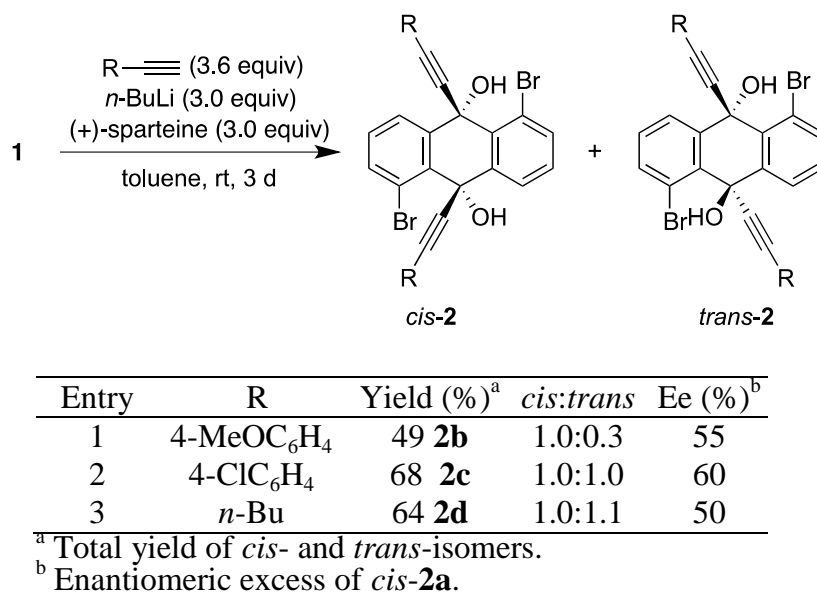


Next we transformed the obtained cis-diol 2a into chiral triptycenes 3 via a Ru-catalyzed [2+2+2] cycloaddition with alkynes (Table 4). ${ }^{11}$ Excess amounts of alkynes were needed, but chiral triptycenes were obtained by the reaction with hex-1-yne, oct-1-yne and 1,4-dihydroxybut-2-yne. The retention of the enantiomeric excess of the obtained cycloadducts was determined for each entry.

Table 4. Ru-catalyzed stereospecific $[2+2+2]$ cycloaddition of cis-2a with alkynes

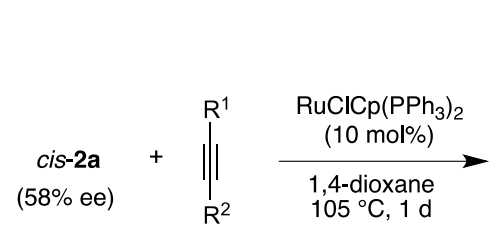

(10 equiv)

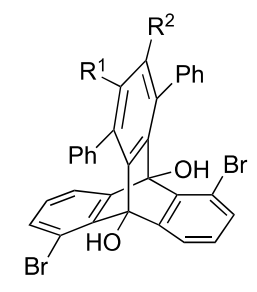

$3(58 \%$ ee $)$

\begin{tabular}{cccc}
\hline Entry & $\mathrm{R}^{1}$ & $\mathrm{R}^{2}$ & Yield (\%) \\
\hline 1 & $n-\mathrm{C}_{4} \mathrm{H}_{9}$ & $\mathrm{H}$ & 59 3aa \\
2 & $n-\mathrm{C}_{6} \mathrm{H}_{13}$ & $\mathrm{H}$ & 53 3ab \\
3 & $\mathrm{CH}_{2} \mathrm{OH}$ & $\mathrm{CH}_{2} \mathrm{OH}$ & 51 3ac \\
\hline
\end{tabular}

Finally, we demonstrated the use of the obtained triptycene as a chiral building block. Consecutive Suzuki coupling of dibromide 3ac proceeded in high yield and chiral diarylsubstituted triptycene $\mathbf{4}$ was obtained (Eq. 1).

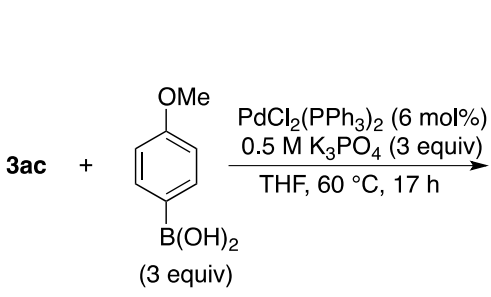

\section{Conclusion}

We have developed the first example of an asymmetric synthesis of chiral triptycenes via an enantioselective protocol. Consecutive alkynylation of 1,5-dibromoanthracene-9,10-dione using sparteine-based chiral alkynyllithium reagents along with Ru-catalyzed $[2+2+2]$ cycloaddition with alkynes gave chiral triptycenes with moderate enantiomeric excesses. The obtained dibromo products can be used as chiral building blocks for the construction of more complex molecules.

\section{Experimental}

\subsection{General}

Anhydrous toluene was purchased and dried over activated molecular sieves $4 \AA$. All reactions were carried out under an argon atmosphere in oven-dried glassware with a magnetic stirring bar. 1,5-Dibromoanthracene-9,10-dione 1 was prepared by a literature method. ${ }^{17}$ NMR spectra were recorded with a JEOL AL-500 (500 MHz) using tetramethylsilane as an internal standard and $\mathrm{CDCl}_{3}$ or acetone- $d_{6}$. High-resolution mass spectra (HRMS) were measured on a Thermo Scientific Exactive Plus orbitrap mass spectrometer. Optical rotations were measured on a JASCO DIP-1000 polarimeter. Ee was determined by HPLC analysis with detection at $254 \mathrm{~nm}$ using Daicel chiral columns $(4.6 \times 250 \mathrm{~mm})$ at room temperature.

\subsection{Typical experimental procedure for the enantioselective alkynylation (entry 1 in Table 2)}

A solution of $n$-butyllithium (1.6 M in hexane, $0.095 \mathrm{~mL}$, $0.15 \mathrm{mmol})$ and $(+)$-sparteine $(34.5 \mu \mathrm{L}, 0.15 \mathrm{mmol})$ was added dropwise to a toluene solution $(0.3 \mathrm{~mL})$ of phenylacetylene $(19.8$ $\mu \mathrm{L}, 0.18 \mathrm{mmol})$ at $0{ }^{\circ} \mathrm{C}$. The reaction mixture was stirred for one hour at room temperature. 1,5-Dibromoanthracene-9,10-dione $\mathbf{1}$ (18.3 $\mathrm{mg}, 0.05 \mathrm{mmol}$ ) was then added to the solution and the reaction mixture was stirred for 3 days. The reaction was quenched with saturated $\mathrm{NH}_{4} \mathrm{Cl}$ aqueous solution, and the organic materials were extracted with ethyl acetate. The combined organic layer was washed with water and brine, then dried over $\mathrm{Na}_{2} \mathrm{SO}_{4}$. After the solvent was removed under reduced pressure, the resulting crude products were purified by preparative TLC, and analytically pure cis-2a $(6.2 \mathrm{mg}, 0.010$ $\mathrm{mmol})$ and trans-2a (21.3 $\mathrm{mg}, 0.037 \mathrm{mmol})$ were obtained.

\subsection{1. cis-1,5-Dibromo-9,10-bis(phenylethynyl)- 9,10-dihydroanthracene-9,10-diol cis-2a}

Off-white solid; mp $>150{ }^{\circ} \mathrm{C}$ (dec.); ${ }^{1} \mathrm{H}$ NMR (acetone- $\left.d_{6}\right) \delta$ 5.93 (s, 2H), 7.25-7.37 (m, 10H), 7.44 (dd, $J=8.0,8.0 \mathrm{~Hz}, 2 \mathrm{H})$, $7.80(\mathrm{~d}, J=7.8 \mathrm{~Hz}, 2 \mathrm{H}), 8.22(\mathrm{~d}, J=8.1 \mathrm{~Hz}, 2 \mathrm{H}) ;{ }^{13} \mathrm{C} \mathrm{NMR}$ (acetone- $d_{6}$ ) $\delta 67.0,86.4,92.8,123.2,123.4,129.3,129.5$ 129.9, 130.6, 132.2, 133.4, 136.1, 141.9; HRMS (ESI positive) $\mathrm{m} / \mathrm{z}$ calcd for $\mathrm{C}_{30} \mathrm{H}_{18} \mathrm{Br}_{2} \mathrm{NaO}_{2}\left([\mathrm{M}+\mathrm{Na}]^{+}\right)$: 590.9566. Found: 590.9565. $[\alpha]_{\mathrm{D}}^{26}=-128\left(c 0.7, \mathrm{CHCl}_{3}\right) 58 \%$ ee. Ee was determined by HPLC analysis using Daicel Chiralpak IC (eluent: $10 \%$ ethyl acetate in hexane, flow rate: $1.0 \mathrm{~mL} / \mathrm{min}$, retention time: 22.0 min for major isomer and $27.8 \mathrm{~min}$ for minor isomer).

4.2.2. $\operatorname{trans}-1,5-D i b r o m o-9,10$ -

bis (phenylethynyl) -9,10-dihydroanthracene-9,10diol trans-2a

Off-white solid; mp $>150{ }^{\circ} \mathrm{C}$ (dec.); ${ }^{1} \mathrm{H}$ NMR (acetone- $\left.d_{6}\right) \delta$ $5.88(\mathrm{~s}, 2 \mathrm{H}), 7.32-7.42(\mathrm{~m}, 10 \mathrm{H}), 7.44(\mathrm{dd}, J=8.0,8.0 \mathrm{~Hz}, 2 \mathrm{H})$, $7.81(\mathrm{~d}, J=7.9 \mathrm{~Hz}, 2 \mathrm{H}), 8.25(\mathrm{~d}, J=8.2 \mathrm{~Hz}, 2 \mathrm{H}) ;{ }^{13} \mathrm{C}$ NMR (acetone- $d_{6}$ ) $\delta 67.7,86.9,93.8,123.5,123.7,129.4,129.6$, 130.6, 130.7, 132.3, 133.8, 136.3, 142.4; HRMS (ESI positive) $\mathrm{m} / \mathrm{z}$ calcd for $\mathrm{C}_{30} \mathrm{H}_{18} \mathrm{Br}_{2} \mathrm{NaO}_{2}\left([\mathrm{M}+\mathrm{Na}]^{+}\right):$590.9566. Found: 590.9567

4.2.3. cis -1,5-Dibromo-9,10-dihydro-9, 10 - bis [2 (4-methoxyphenyl)ethynyl]anthracene-9,10-diol cis - $2 \mathrm{~b}$

Yellow solid; mp $>150{ }^{\circ} \mathrm{C}$ (dec.); ${ }^{1} \mathrm{H}$ NMR (acetone- $\left.d_{6}\right) \delta$ $3.77(\mathrm{~s}, 6 \mathrm{H}), 5.82(\mathrm{~s}, 2 \mathrm{H}), 6.84(\mathrm{~d}, J=9.0 \mathrm{~Hz}, 4 \mathrm{H}), 7.28(\mathrm{~d}, J=$ $9.0 \mathrm{~Hz}, 4 \mathrm{H}), 7.42(\mathrm{dd}, J=8.0,8.0 \mathrm{~Hz}, 2 \mathrm{H}), 7.78(\mathrm{~d}, J=7.9 \mathrm{~Hz}$, $2 \mathrm{H}), 8.20(\mathrm{~d}, J=8.3 \mathrm{~Hz}, 2 \mathrm{H}) ;{ }^{13} \mathrm{C}$ NMR (acetone- $\left.d_{6}\right) \delta 55.6$, 67.0, 86.5, 91.4, 114.8, 115.4, 123.1, 129.9, 130.4, 133.5, 133.8, 136.0, 142.0, 160.9; HRMS (ESI positive) $\mathrm{m} / \mathrm{z}$ calcd for $\mathrm{C}_{32} \mathrm{H}_{22} \mathrm{Br}_{2} \mathrm{NaO}_{4}\left([\mathrm{M}+\mathrm{Na}]^{+}\right): 650.9777$. Found: 650.9772. $[\alpha]_{\mathrm{D}}^{26}=-$ 112 (c $\left.0.67, \mathrm{CHCl}_{3}\right) 55 \%$ ee. Ee was determined by HPLC analysis using Daicel Chiralpak IE-3 (eluent: $20 \%$ ethyl acetate in hexane, flow rate: $1.0 \mathrm{~mL} / \mathrm{min}$, retention time: $20.2 \mathrm{~min}$ for major isomer and $16.9 \mathrm{~min}$ for minor isomer).

4.2.4. $\operatorname{trans}-1,5$-Dibromo-9,10-dihy dro-9,10bis [ 2 - (4-methoxyphenyl)ethynyl]anthracene 9,10-diol trans-2b

Pale orange solid; $\mathrm{mp}>150{ }^{\circ} \mathrm{C}$ (dec.) ${ }^{1} \mathrm{H}$ NMR (acetone- $\left.d_{6}\right) \delta$ $3.80(\mathrm{~s}, 6 \mathrm{H}), 5.78(\mathrm{~s}, 2 \mathrm{H}), 6.90(\mathrm{~d}, J=9.1 \mathrm{~Hz}, 4 \mathrm{H}), 7.33(\mathrm{~d}, J=$ $9.0 \mathrm{~Hz}, 4 \mathrm{H}), 7.42(\mathrm{dd}, J=8.0,8.0 \mathrm{~Hz}, 2 \mathrm{H}), 7.79(\mathrm{~d}, J=7.8 \mathrm{~Hz}$, $2 \mathrm{H}), 8.23(\mathrm{~d}, J=8.2 \mathrm{~Hz}, 2 \mathrm{H}) ;{ }^{13} \mathrm{C}$ NMR (acetone- $\left.d_{6}\right) \delta 55.8$, 


\section{Tetrahedron}

67.7, 87.0, 92.5, 115.1, 115.7, 123.5, 130.6, 130.6, 133.9, 134.0, 136.2, 161.1; HRMS (ESI positive) $\mathrm{m} / \mathrm{z}$ calcd for $\mathrm{C}_{32} \mathrm{H}_{22} \mathrm{Br}_{2} \mathrm{NaO}_{2}\left([\mathrm{M}+\mathrm{Na}]^{+}\right): 650.9777$. Found: 650.9772 .

4.2.5. cis-1,5-Dibromo-9,10-bis[2-(4chlorophenyl)ethynyl]-9,10-dihydroanthracene9,10-diol cis-2c

Pale yellow solid; $\mathrm{mp}>150{ }^{\circ} \mathrm{C}$ (dec.); ${ }^{1} \mathrm{H}$ NMR (acetone- $\left.d_{6}\right) \delta$ $5.93(\mathrm{~s}, 2 \mathrm{H}), 7.33-7.38(\mathrm{~m}, 8 \mathrm{H}), 7.44(\mathrm{dd}, J=8.0,8.0 \mathrm{~Hz}, 2 \mathrm{H})$, $7.81(\mathrm{~d}, J=7.8 \mathrm{~Hz}, 2 \mathrm{H}), 8.20(\mathrm{~d}, J=8.2 \mathrm{~Hz}, 2 \mathrm{H}) ;{ }^{13} \mathrm{C} \mathrm{NMR}$ (acetone- $d_{6}$ ) $\delta ; 67.1,85.4,93.9,122.3,123.4,129.7,130.1$, 130.9, 133.4, 134.0, 135.2, 136.4, 141.9; HRMS (ESI positive) $\mathrm{m} / \mathrm{z}$ calcd for $\mathrm{C}_{30} \mathrm{H}_{16} \mathrm{Br}_{2} \mathrm{Cl}_{2} \mathrm{NaO}_{2}\left([\mathrm{M}+\mathrm{Na}]^{+}\right)$: 658.8786 . Found: 658.8784. $[\alpha]_{\mathrm{D}}^{26}=-119\left(c \quad 0.37, \mathrm{CHCl}_{3}\right) 60 \%$ ee. Ee was determined by HPLC analysis using Daicel Chiralpak IF-3 (eluent: $20 \%$ ethyl acetate in hexane, flow rate: $1.0 \mathrm{~mL} / \mathrm{min}$, retention time: $16.2 \mathrm{~min}$ for major isomer and $14.3 \mathrm{~min}$ for minor isomer).

4.2.6. trans-1,5-Dibromo-9,10-bis[2-(4chlorophenyl)ethynyl]-9,10-dihydroanthracene9,10-diol trans-2c

Pale yellow solid; mp $>150{ }^{\circ} \mathrm{C}$ (dec.); ${ }^{1} \mathrm{H}$ NMR $\left(\mathrm{CDCl}_{3}\right) \delta$ $4.61(\mathrm{~s}, 2 \mathrm{H}), 7.25(\mathrm{~d}, J=8.8 \mathrm{~Hz}, 4 \mathrm{H}), 7.30(\mathrm{~d}, J=8.8 \mathrm{~Hz}, 4 \mathrm{H})$, $7.39(\mathrm{dd}, J=8.0,8.0 \mathrm{~Hz}, 2 \mathrm{H}), 7.76(\mathrm{~d}, J=8.0 \mathrm{~Hz}, 2 \mathrm{H}), 8.17$ (d, $J=8.0 \mathrm{~Hz}, 2 \mathrm{H}) ;{ }^{13} \mathrm{C} \mathrm{NMR}\left(\mathrm{CDCl}_{3}\right) \delta 67.4,86.3,92.8,120.9$, $121.9,128.8,130.1,130.3,132.6,133.0,135.0,135.6,139.2$; HRMS (ESI positive) $\mathrm{m} / \mathrm{z}$ calcd for $\mathrm{C}_{30} \mathrm{H}_{16} \mathrm{Br}_{2} \mathrm{Cl}_{2} \mathrm{NaO}_{2}$ $\left([\mathrm{M}+\mathrm{Na}]^{+}\right)$: 658.8786. Found: 658.8779 .

\subsection{7. cis -1,5-Dibromo-9, $10-\mathrm{di}($ hex - $1-\mathrm{ynyl})-9,10$ -} dihydroanthracene $-9,10-d i o l$ cis $-2 d$

Pale yellow solid; mp 75-78 ${ }^{\circ} \mathrm{C} ;{ }^{1} \mathrm{H}$ NMR $\left(\mathrm{CDCl}_{3}\right) \delta 0.84(\mathrm{t}, J$ $=7.4 \mathrm{~Hz}, 6 \mathrm{H}), 1.27-1.37(\mathrm{~m}, 4 \mathrm{H}), 1.39-1.48(\mathrm{~m}, 4 \mathrm{H}), 2.14(\mathrm{t}, J$ $=7.2, \mathrm{~Hz}, 4 \mathrm{H}), 4.53(\mathrm{~s}, 2 \mathrm{H}), 7.30(\mathrm{dd}, J=8.0,8.0 \mathrm{~Hz}, 2 \mathrm{H}), 7.67$ $(\mathrm{d}, J=7.9 \mathrm{~Hz}, 2 \mathrm{H}), 8.05(\mathrm{~d}, J=8.2 \mathrm{~Hz}, 2 \mathrm{H}) ;{ }^{13} \mathrm{C} \mathrm{NMR}\left(\mathrm{CDCl}_{3}\right)$ $\delta 13.7,18.8,22.1,30.4,66.2,82.4,88.2,121.4,129.3,129.7$, 132.7, 135.1, 139.7; HRMS (ESI positive) $\mathrm{m} / \mathrm{z}$ calcd for $\mathrm{C}_{26} \mathrm{H}_{26} \mathrm{Br}_{2} \mathrm{NaO}_{2}\left([\mathrm{M}+\mathrm{Na}]^{+}\right): 551.0192$. Found: 551.0195. $[\alpha]_{\mathrm{D}}^{26}=-$ $87.2\left(c 0.62, \mathrm{CHCl}_{3}\right) 50 \%$ ee. Ee was determined by HPLC analysis using Daicel Chiralpak IC (eluent: $10 \%$ ethyl acetate in hexane, flow rate: $1.0 \mathrm{~mL} / \mathrm{min}$, retention time: $14.8 \mathrm{~min}$ for major isomer and $17.5 \mathrm{~min}$ for minor isomer).

4.2.8. trans -1,5-Dibromo-9,10-di(hex-1-ynyl)9,10-dihydroanthracene-9,10-diol trans-2d

Pale pink solid; mp 176-178 ${ }^{\circ} \mathrm{C} ;{ }^{1} \mathrm{H}$ NMR $\left(\mathrm{CDCl}_{3}\right) \delta 0.85(\mathrm{t}$, $J=7.4 \mathrm{~Hz}, 6 \mathrm{H}), 1.28-1.38(\mathrm{~m}, 4 \mathrm{H}), 1.39-1.48(\mathrm{~m}, 4 \mathrm{H}), 2.14(\mathrm{t}, J$ $=7.1 \mathrm{~Hz}, 4 \mathrm{H}), 4.40(\mathrm{~s}, 2 \mathrm{H}), 7.30(\mathrm{dd}, J=8.0,8.0 \mathrm{~Hz}, 2 \mathrm{H}), 7.68$ $(\mathrm{d}, J=7.9 \mathrm{~Hz}, 2 \mathrm{H}), 8.06(\mathrm{~d}, J=8.2 \mathrm{~Hz}, 2 \mathrm{H}) ;{ }^{13} \mathrm{C} \mathrm{NMR}\left(\mathrm{CDCl}_{3}\right)$ $\delta 13.7,18.8,22.1,30.3,67.0,83.3,88.9,121.7,129.7,129.9$, 133.0, 135.2, 139.9; HRMS (ESI positive) $\mathrm{m} / \mathrm{z}$ calcd for $\mathrm{C}_{26} \mathrm{H}_{26} \mathrm{Br}_{2} \mathrm{NaO}_{2}\left([\mathrm{M}+\mathrm{Na}]^{+}\right)$: 551.0192. Found: 551.0195.

\subsection{Typical experimental procedure for the $[2+2+2]$ cycloaddition (entry 1 in Table 4)}

At first, $\mathrm{RuClCp}\left(\mathrm{PPh}_{3}\right)_{2}(3.6 \mathrm{mg}, 0.005 \mathrm{mmol} 10 \mathrm{~mol} \%)$ and cis-2a $(28.5 \mathrm{mg}, 0.05 \mathrm{mmol})$ were placed in a Schlenk tube, which was then evacuated and backfilled with argon. 1-Hexyne $(56.8 \mu \mathrm{L}, 0.5 \mathrm{mmol})$ and 1,4 -dioxane $(0.5 \mathrm{~mL})$ were then added and the reaction vessel was immersed in an oil bath at $105{ }^{\circ} \mathrm{C}$ for one day. The solvent was removed under reduced pressure, and the crude products were purified by preparative TLC to give analytically pure 3aa (14.7 mg, $0.029 \mathrm{mmol}, 59 \%)$.

4.3.1. 5,13-Dibromo-2-butyl-1,4-diphenyl-9,10dihydro-9, $10-[1,2]$ benzenoanthracene 3 aa
Yellow foam; ${ }^{1} \mathrm{H}$ NMR (acetone- $\left.d_{6}\right) \delta 0.66(\mathrm{t}, J=7.5 \mathrm{~Hz}$, $3 \mathrm{H}), 0.82-0.97(\mathrm{~m}, 2 \mathrm{H}), 1.03-1.12(\mathrm{~m}, 2 \mathrm{H}), 2.15-2.31(\mathrm{~m}, 2 \mathrm{H})$, $3.49(\mathrm{~s}, 1 \mathrm{H}), 4.05(\mathrm{~s}, 1 \mathrm{H}), 6.86(\mathrm{~s}, 1 \mathrm{H}), 6.97-7.10(\mathrm{~m}, 2 \mathrm{H}), 7.30$ $(\mathrm{dd}, J=8.7,8.7 \mathrm{~Hz}, 2 \mathrm{H}), 7.36(\mathrm{~d}, J=8.0 \mathrm{~Hz}, 1 \mathrm{H}), 7.38-7.67$ $(\mathrm{m}, 11 \mathrm{H}) ;{ }^{13} \mathrm{C}$ NMR (acetone- $\left.d_{6}\right) \delta 14.0,23.3,33.0,34.3,82.0$, $82.4,114.7,114.9,121.8,122.1,128.3,128.5,128.8,129.4$, $129.6,130.2,131.0,131.4,133.7,133.7,136.2,137.2,140.3$, $140.3,140.4,140.6,140.6,143.0,143.1,150.3,150.4$ (a pair of peaks at aromatic region is overlapped); HRMS (ESI positive) $\mathrm{m} / \mathrm{z}$ calcd for $\mathrm{C}_{36} \mathrm{H}_{28} \mathrm{Br}_{2} \mathrm{NaO}_{2}\left([\mathrm{M}+\mathrm{Na}]^{+}\right): 673.0348$. Found: 673.0345. $[\alpha]_{\mathrm{H} g 435}^{26}=-212\left(c \quad 0.20, \mathrm{CHCl}_{3}\right) \quad 58 \%$ ee. Ee was determined by HPLC analysis using Daicel Chiralpak IA (eluent: $2 \%$ dichloromethane in hexane, flow rate: $1.0 \mathrm{~mL} / \mathrm{min}$, retention time: $7.4 \mathrm{~min}$ for major isomer and $10.5 \mathrm{~min}$ for minor isomer).

\subsubsection{5,13-Dibromo-2-hexyl-1,4-diphenyl-9,10-} dihydro-9,10-[1,2]benzenoanthracene $3 \mathrm{ab}$

Tan colored foam; ${ }^{1} \mathrm{H}$ NMR (acetone- $\left.d_{6}\right) \delta 0.75(\mathrm{t}, J=7.3$ $\mathrm{Hz}, 3 \mathrm{H}), 0.83-0.95$ (m, 2H), 0.99-1.17 (m, 6H), 2.14-2.31 (m, $2 \mathrm{H}), 3.49(\mathrm{~s}, 1 \mathrm{H}), 4.04(\mathrm{~s}, 1 \mathrm{H}), 6.86(\mathrm{~s}, 1 \mathrm{H}), 6.99-7.09(\mathrm{~m}, 2 \mathrm{H})$, 7.27-7.66 (m, 14H); ${ }^{13} \mathrm{C}$ NMR (acetone- $\left.d_{6}\right) \delta 14.4,23.1,32.1$, $32.1,33.4,82.0,82.4,114.7,114.9,121.8,122.1,128.3,128.5$, $128.8,129.4,129.6,130.2,131.0,131.4,133.7,133.7,136.2$, $137.2,140.3,140.4,140.4,140.6,140.6,143.0,143.1,150.3$, 150.4 (a pair of peaks at aliphatic region and a pair of peaks at aromatic region are overlapped); HRMS (ESI positive) $\mathrm{m} / \mathrm{z}$ calcd for $\mathrm{C}_{38} \mathrm{H}_{32} \mathrm{Br}_{2} \mathrm{NaO}_{2}\left([\mathrm{M}+\mathrm{Na}]^{+}\right)$: 701.0661. Found: 701.0657. $[\alpha]_{\mathrm{H} 433}^{26}=-216\left(c \quad 0.21, \mathrm{CHCl}_{3}\right) 58 \%$ ee. Ee was determined by HPLC analysis using Daicel Chiralpak IA (eluent: $2 \%$ dichloromethane in hexane, flow rate: $1.0 \mathrm{~mL} / \mathrm{min}$, retention time: $7.1 \mathrm{~min}$ for major isomer and $10.1 \mathrm{~min}$ for minor isomer).

4.3.3. 5,13-Dibromo-2,3-bis(hydroxymethyl) - 1,4diphenyl-9,10-dihydro-9,10-

$[1,2]$ benzenoanthracene-9,10-diol 3ac

Pale yellow foam; ${ }^{1} \mathrm{H}$ NMR (acetone- $\left.d_{6}\right) \delta 3.49$ (s, 2H), 4.08$4.13(\mathrm{~m}, 2 \mathrm{H}), 4.23-4.31(\mathrm{~m}, 2 \mathrm{H}), 4.38-4.45(\mathrm{dd}, J=7.9,7.9 \mathrm{~Hz}$, 2H), 7.01-7.08 (m, 2H), 7.27-7.36 (m, 4H), 7.43-7.49 (m, 2H), 7.54-7.66 (m, 8H); ${ }^{13} \mathrm{C}$ NMR $\delta 59.4,82.1,114.8,122.0,128.4$, $128.9,129.3,129.5,130.0,131.8,133.7,137.3,139.0,140.2$, 140.3, 142.7, 150.0; HRMS (ESI positive) $\mathrm{m} / \mathrm{z}$ calcd for $\mathrm{C}_{34} \mathrm{H}_{24} \mathrm{Br}_{2} \mathrm{NaO}_{4}\left([\mathrm{M}+\mathrm{Na}]^{+}\right)$: 676.9934. Found: 676.9935. $[\alpha]_{\mathrm{Hg} 435}^{26}=$ $-17.0\left(c 0.85, \mathrm{CHCl}_{3}\right) 58 \%$ ee. Ee was determined by HPLC analysis using Daicel Chiralpak IA (eluent: $40 \%$ dichloromethane in hexane, flow rate: $1.0 \mathrm{~mL} / \mathrm{min}$, retention time: $18.1 \mathrm{~min}$ for major isomer and $20.8 \mathrm{~min}$ for minor isomer).

\subsection{Suzuki coupling of 3ac (Eq. 1)}

To a THF solution $(0.1 \mathrm{~mL})$ of dibromo 3ac $(11.2 \mathrm{mg}, 0.017$ $\mathrm{mmol})$, 4-methoxyphenylboronic acid $(7.7 \mathrm{mg}, 0.051 \mathrm{mmol})$, $\mathrm{PdCl}_{2}\left(\mathrm{PPh}_{3}\right)_{2}(1.0 \mathrm{mg}, 0.0014 \mathrm{mmol}, 8.3 \mathrm{~mol} \%)$ and $0.5 \mathrm{M}$ $\mathrm{K}_{3} \mathrm{PO}_{4}$ aq. $(0.1 \mathrm{~mL}, 0.05 \mathrm{mmol})$ were added at room temperature. The mixture was stirred at $60{ }^{\circ} \mathrm{C}$ for $17 \mathrm{~h}$ and then filtered through a pad of silica gel with ethyl acetate. Purification by preparative TLC gave analytically pure product 4 (10.6 mg, $0.015 \mathrm{mmol}, 88 \%)$.

\subsubsection{2,3-Bis(hydroxymethyl) -5,13-bis (4- methoxyphenyl) - 1,4-diphenyl-9,10-dihydro-9,10- $[1,2]$ benzenoanthracene $-9,10-$ diol 4}

Pale yellow foam; ${ }^{1} \mathrm{H}$ NMR $\left(\mathrm{CDCl}_{3}\right) \delta 2.03(\mathrm{~s}, 2 \mathrm{H}), 2.42(\mathrm{~s}$, $2 \mathrm{H}), 3.87(\mathrm{~s}, 6 \mathrm{H}), 4.30(\mathrm{~s}, 4 \mathrm{H}), 6.83(\mathrm{~d}, J=7.7 \mathrm{~Hz}, 4 \mathrm{H}), 6.92(\mathrm{~d}$, $J=8.7 \mathrm{~Hz}, 2 \mathrm{H}), 7.07(\mathrm{dd}, J=7.6,7.6 \mathrm{~Hz}, 2 \mathrm{H}), 7.11-7.17(\mathrm{~m}$, $4 \mathrm{H})$, 7.20-7.25 (m, 4H), 7.27-7.34 (m, 4H), 7.35-7.39 (m, 2H), $7.54(\mathrm{~d}, J=7.7 \mathrm{~Hz}, 2 \mathrm{H}) ;{ }^{13} \mathrm{C} \mathrm{NMR}\left(\mathrm{CDCl}_{3}\right) \delta 55.4,59.5,81.9$, 
$113.1,113.4,120.2,124.9,127.2,128.1,128.2,129.9,130.0$, $130.3,131.5,133.0,135.5,136.1,136.6,139.6,140.0,143.6$, 146.6, 158.8; HRMS (ESI positive) $\mathrm{m} / \mathrm{z}$ calcd for $\mathrm{C}_{48} \mathrm{H}_{38} \mathrm{NaO}_{6}$ $\left([\mathrm{M}+\mathrm{Na}]^{+}\right): 733.2561$. Found: 733.2559. $[\alpha]_{\mathrm{H} g 435}^{26}=23.4(c \quad 0.49$, $\left.\mathrm{CHCl}_{3}\right)$.

\section{Acknowledgments}

This work was supported by ACT-C from JST (Japan) and Waseda University Grant for Special Research Projects (No. 2014B-286). We also thank Mr. Ryotaro Takayama (Waseda Univ.) for his assistance in the preliminary work.

\section{References}

1. Bartlett, P. D.; Ryan, M. J.; Cohen, S. G. J. Am. Chem. Soc. 1942, 64, 2649-2653.

2. Wittig, G.; Ludwig, R. Angew. Chem. 1956, 68, 40-40.

3. O-ki, M. Acc. Chem. Res. 1990, 23, 351-356.

4. For a recent review, see: Jiang, Y.; Chen, C.-F. Eur. J. Org. Chem. 2011, 6377-6403.

5. Sundermeier, M.; Zapf, A.; Mutyala, S.; Baumann, W.; Sans, J.; Weiss, S.; Beller, M. Chem. Eur. J. 2003, 9, 1828-1836.

6. Godinez, C. E.; Zepeda, G.; Garcia-Garibay, M. A. J. Am. Chem. Soc. 2002, 124, 4701-4707.

7. Zong, Q.-S.; Chen, C.-F. Org. Lett. 2006, 8, 211-214.

8. Pochorovski, I.; Diederich, F. Acc. Chem. Res. 2014, 47, 20962105.

9. Swager, T. M. Acc. Chem. Res. 2008, 41, 1181-1189.

10. (a) Sonoda, A.; Ogura, F.; Nakagawa, M. Bull. Chem. Soc. Jpn. 1962, 35, 853-857; (b) de Wit, J.; Wynberg, H. Tetrahedron 1972, 28, 4617-4624.

11. (a) Taylor, M. S.; Swager, T. M. Org. Lett. 2007, 9, 3695-3696. (b) Veller, B. V.; Robinson, D.; Swager, T. M. Angew. Chem. Int. Ed. 2012, 51, 1182-1186.

12. Enantioselective alkynylations of aldehydes using alkynyllithiums: (a) Jiang, B.; Feng, Y. Tetrahedron Lett. 2002, 43, 2975-2977; (b) Tanaka, K.; Kukita, K.;
Ichibakase, T; Kotani, S.; Nakajima, M. Chem. Commun. 2011, 47, 5614-5616.

13. For examples of enantioselective alkynylations of aldehydes using alkynyl silanes, see: (a) Ueda, T.; Tanaka, K.; Ichibakase, T.; Orito, Y.; Nakajima, M. Tetrahedron 2010, 66, 7726-7731; (b) Tanaka, K.; Ueda, T.; Ichibakase, T.; Nakajima, M. Tetrahedron Lett. 2010, 51, 2168-2169.

14. For examples of enantioselective alkynylations of aldehydes using alkynyl zincs, see: (a) Tan, L.; Chen, C. Y.; Tillyer, R. D.; Grabowski, E. J. J.; Reider, P. J. Angew. Chem. Int. Ed. 1999, 38,711-713; (b) Cozzi, P. G. Angew. Chem. Int. Ed. 2003, 42, 2895-2898; (c) Lu, G.; Li, X.; Jia, X.; Chan, W. L.; Chan, A. S. C. Angew. Chem. Int. Ed. 2003, 42, 5057-5058; (d) Liu, L.; Kang, Y. F.; Wang, R.; Zhou, Y. F.; Chen, C.; Ni, M.; Gong, M. Z. Tetrahedron: Asymmetry 2004, 15, 3757-3761; (e) Zhou, Y.; Wang, R.; Xu, Z.; Yan, W.; Liu, L.; Kang, Y.; Han, Z. J. Org. Lett. 2004, 6, 4147-4149; (f) Kang, Y. F.; Liu, L.; Wang, R.; Zhou, Y. F.; Yan, W. J. Adv. Synth. Catal. 2005, 347, 243247; (g) Liu, L.; Wang, R.; Kang, Y. F.; Chen, C.; Xu, Z. Q.; Zhou, Y. F.; Ni, M.; Cai, H. Q.; Gong, M. Z. J. Org. Chem. 2005, 70, 1084-1086; (h) Ni, M.; Wang, R.; Han, Z. J.; Mao, B.; Da, C. S.; Liu, L.; Chen, C. Adv. Synth. Catal. 2005, 347, 1659-1665; (i) Wang, S. H.; Tu, Y. Q.; Chen, P. Chin. J. Chem. 2006, 24, 165168; (j) Lu, G.; Li, X.; Li, Y. M.; Kwong, F. Y.; Chan, A. S. C. Adv. Synth. Catal. 2006, 348, 1926-1933; (k) Ding, J.; Shen, Z. X.; Luo, X. Q.; Chen, W. Y.; Zhang, Y. W. Chin. J. Chem. 2006, 24, 1285-1289; (1) Ni, M.; Zhou, Y. F.; Chen, C.; Xu, J. K.; Wang, R. Chin. J. Chem. 2007, 25, 694-697; (m) Chen, C.; Hong, L.; Zhang, B.; Wang, R. Tetrahedron: Asymmetry 2008, 19, 191-196; (n) Li, H.; Li, X.; Xue, F.; Huang, Y.; Jin, W.; Wan, B. Chin. J. Chem. 2009, 27, 2013-2019; (o) Zhang, G. W.; Meng, W.; Ma, H.; Nie, J.; Zhang, W. Q.; Ma, J. A. Angew. Chem. Int. Ed. 2011, 50, 3538-3542.

15. For examples of enantioselective alkynylations of ketones, see: (a) Liu, L.; Wang, R.; Kang, Y. F.; Cai, H. Q.; Chen, C. Synlett 2006, 1245-1249; (b) Dhayalan, V.; Murakami, R.; Hayashi, M. Asian J. Chem. 2013, 25, 7505-7508.

16. When 1,5-dichloroanthracene-9,10-dione was used in place of dibromo 1 under the reaction conditions of entry 1 in Table 2, the ee of the obtained dialkylated product was $37 \%$ ee.

17. Seidel, N.; Hahn, T.; Liebing, S.; Seichter, W.; Kortus, J.; Weber, E. New J. Chem. 2013, 37, 601-610. 


\section{Graphical Abstract}

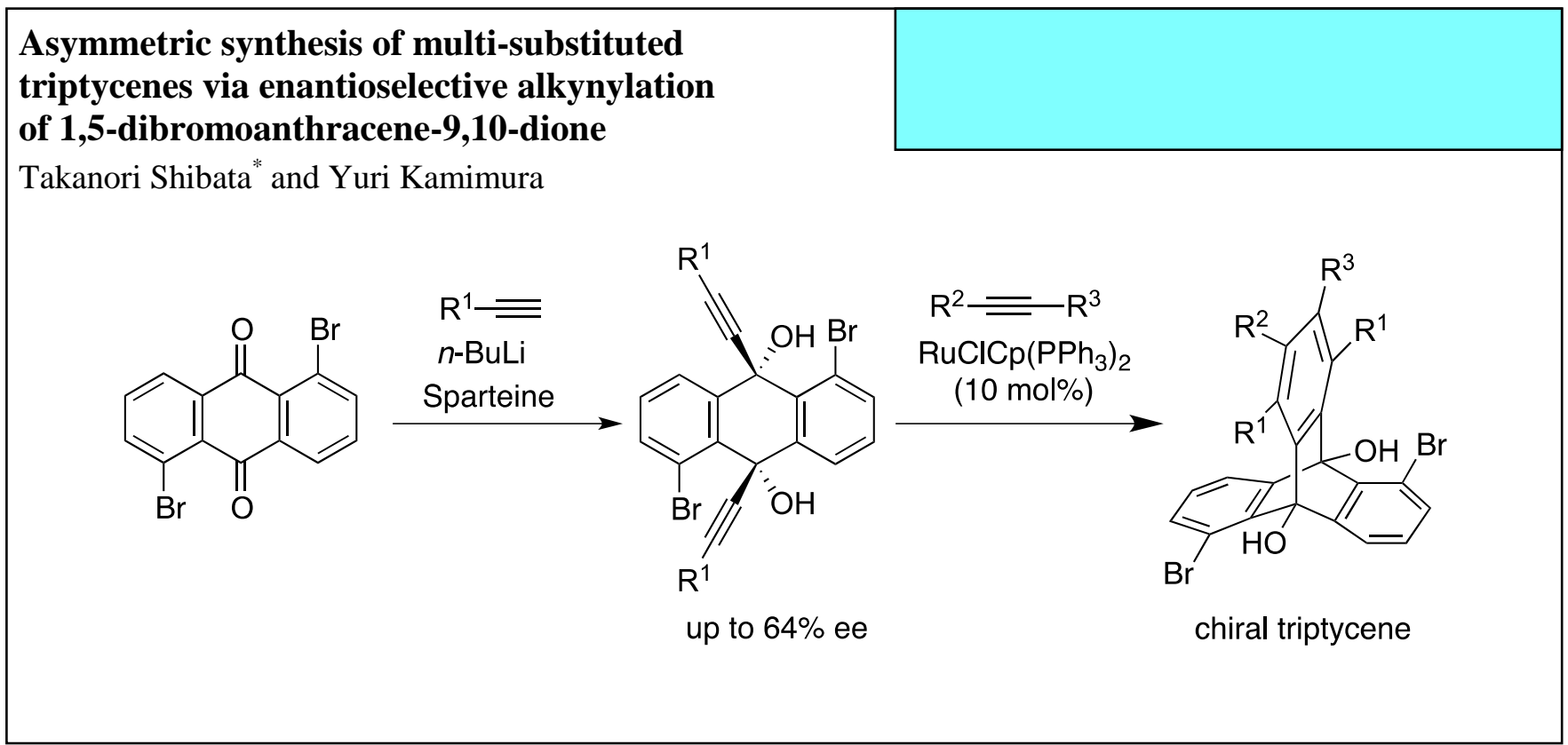




\section{Stereochemistry Abstract}

To create your abstract, type over the instructions in the template box below.

Fonts or abstract dimensions should not be changed or altered. You may insert more abstracts by copying this box or by using the menu option to insert a stereochemistry abstract.

Takanori Shibata and Yuri Kamimura

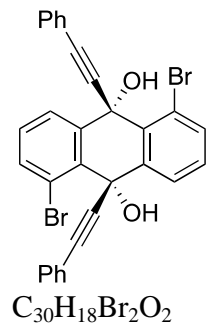

$\mathrm{Ee}=58 \%$ from HPLC

$[\alpha]_{\mathrm{D}}^{26}=-128\left(c 0.70, \mathrm{CHCl}_{3}\right)$

Source of chirality: (+)-sparteine

Absolute configuration: not determined

$\mathrm{C}_{30} \mathrm{H}_{18} \mathrm{Br}_{2} \mathrm{O}_{2}$

cis-1,5-Dibromo-9,10-bis(phenylethynyl)-9,10-dihydroanthracene-9,10-diol

Takanori Shibata and Yuri Kamimura

$4-\mathrm{MeOC}_{6} \mathrm{H}_{4}$

$\mathrm{Ee}=55 \%$ from HPLC

$[\alpha]_{\mathrm{D}}^{26}=-112\left(c 0.67, \mathrm{CHCl}_{3}\right)$

Source of chirality: (+)-sparteine

Absolute configuration: not determined

4- $\mathrm{MeOC}_{6} \mathrm{H}_{4}$

$\mathrm{C}_{32} \mathrm{H}_{22} \mathrm{Br}_{2} \mathrm{O}_{2}$

cis-1,5-Dibromo-9,10-dihydro-9,10-bis[2-(4-methoxyphenyl)ethynyl]anthracene-9,10-diol

Takanori Shibata and Yuri Kamimura

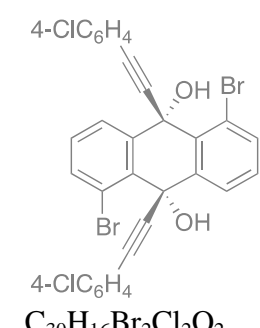

$\mathrm{Ee}=60 \%$ from HPLC

$[\alpha]_{\mathrm{D}}{ }^{26}=-119\left(c 0.37, \mathrm{CHCl}_{3}\right)$

Source of chirality: (+)-sparteine

Absolute configuration: not determined

$\mathrm{C}_{30} \mathrm{H}_{16} \mathrm{Br}_{2} \mathrm{Cl}_{2} \mathrm{O}_{2}$

cis-1,5-Dibromo-9,10-bis(2-(4-chlorophenyl)ethynyl)-9,10-dihydroanthracene-9,10-diol 
Takanori Shibata and Yuri Kamimura

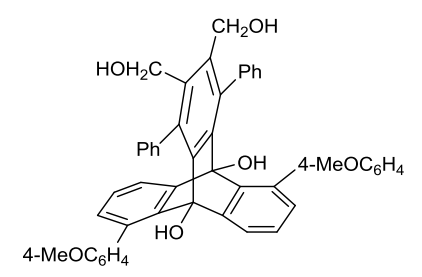

$[\alpha]_{\mathrm{Hg} 435}{ }^{26}=+23.4\left(c 0.49, \mathrm{CHCl}_{3}\right)$

Source of chirality: (+)-sparteine

Absolute configuration: not determined

$\mathrm{C}_{48} \mathrm{H}_{38} \mathrm{O}_{6}$

2,3-Bis(hydroxymethyl)-5,13-bis(4-methoxyphenyl)-1,4-diphenyl-9,10-dihydro-9,10-[1,2]benzenoanthracene-9,10-diol 
Takanori Shibata and Yuri Kamimura

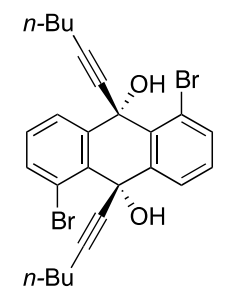

$\mathrm{Ee}=50 \%$ from HPLC

$[\alpha]_{\mathrm{D}}^{26}=-87.2\left(c 0.62, \mathrm{CHCl}_{3}\right)$

Source of chirality: (+)-sparteine

Absolute configuration: not determined

$\mathrm{C}_{30} \mathrm{H}_{16} \mathrm{Br}_{2} \mathrm{Cl}_{2} \mathrm{O}_{2}$

cis-1,5-Dibromo-9,10-di(hex-1-ynyl)-9,10-dihydroanthracene-9,10-diol

Takanori Shibata and Yuri Kamimura

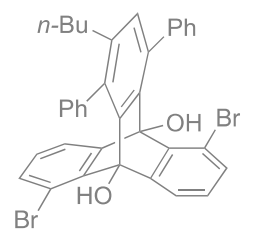

$\mathrm{Ee}=58 \%$ from HPLC

$[\alpha]_{\mathrm{Hg} 435}^{26}=-212\left(c 0.20, \mathrm{CHCl}_{3}\right)$

Source of chirality: (+)-sparteine

Absolute configuration: not determined

$\mathrm{C}_{36} \mathrm{H}_{28} \mathrm{Br}_{2} \mathrm{O}_{2}$

5,13-Dibromo-2-butyl-1,4-diphenyl-9,10-dihydro-9,10-[1,2]benzenoanthracene

Takanori Shibata and Yuri Kamimura

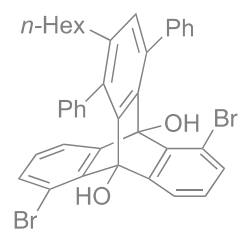

$\mathrm{Ee}=58 \%$ from HPLC

$[\alpha]_{\mathrm{Hg} 435}^{26}=-216\left(c 0.21, \mathrm{CHCl}_{3}\right)$

Source of chirality: (+)-sparteine

$\mathrm{Br}$

Absolute configuration: not determined

$\mathrm{C}_{38} \mathrm{H}_{32} \mathrm{Br}_{2} \mathrm{O}_{2}$

5,13-Dibromo-2-hexyl-1,4-diphenyl-9,10-dihydro-9,10-[1,2]benzenoanthracene

Takanori Shibata and Yuri Kamimura

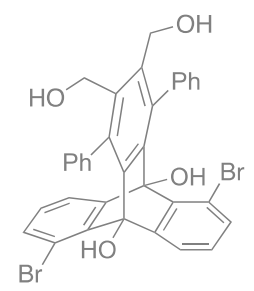

$\mathrm{Ee}=58 \%$ from HPLC

$[\alpha]_{\mathrm{Hg} 435}{ }^{26}=-17.0\left(c 0.85, \mathrm{CHCl}_{3}\right)$

Source of chirality: (+)-sparteine

Absolute configuration: not determined

$\mathrm{C}_{34} \mathrm{H}_{24} \mathrm{Br}_{2} \mathrm{O}_{4}$

5,13-Dibromo-2,3-bis(hydroxymethyl)-1,4-diphenyl-9,10-dihydro-9,10-[1,2]benzenoanthracene-9,10-diol 